\title{
ON THE CONSTRUCTION OF GENERALIZED MEASURE PRESERVING TRANSFORMATIONS WITH GIVEN MARGINALS
}

Theodore P. Hill*

School of Mathematics

Georgia Institute of Technology

Atlanta, GA 30332 USA and

Ulrich Krengel**

Institut für Mathematische Stochastik

Lotzestr. 13, Univ. Göttingen

W-3400 Göttingen, Germany

Abstract

Measure preserving transformations generate stationary processes and vice versa. Which processes $\left(X_{i}\right)$ correspond to the class of generalized measure preserving transformations? We give necessary conditions and show that they are sufficient for 2-valued processes as far as the marginals of $\left(X_{0}, X_{1}, X_{2}, X_{3}\right)$ are concerned. The general problem remains open. Our main tool is a construction of a class of generalized measure preserving transformations which may be of independent interest.

\section{$\S 1$. Introduction}

The notion of generalized measure preserving transformation (gmp-transformation) was introduced in $[\mathrm{K}]$ as a mathematical model for the movement of sets of incompressible objects subject to interaction. Let $(\Omega, \mathcal{A}, \mu)$ be a probability space. A gmptransformation is a map $\phi: \mathcal{A} \rightarrow \mathcal{A}$ which is order preserving and which preserves $\mu$. In other words, $A \subset B$ implies $\phi(A) \subset \phi(B)$, and $\mu(\phi(A))=\mu(A)$ holds for all $A \in \mathcal{A}$.

A measure preserving transformation $\tau: \Omega \rightarrow \Omega$ induces a gmp-transformation $\phi_{\tau}$ by setting $\phi_{\tau}(A)=\tau^{-1} A$. In general, however, a gmp-transformation need not commute with the formation of unions or intersections. We only have

$$
\phi(A \cap B) \subset \phi(A) \cap \phi(B) \quad(A, B \in \mathcal{A})
$$

and

$$
\phi(A \cup B) \supset \phi(A) \cup \phi(B) \quad(A, B \in \mathcal{A}) .
$$

Let $\left(X_{n}\right)_{n \geq 0}$ be a real-valued stochastic process defined on a probability space $\left(\Omega^{\prime}, \mathcal{A}^{\prime}, P\right)$. It is well known that there exists a measure preserving $\tau$ on a suitable probability space $(\Omega, \mathcal{A}, \mu)$ and a measurable $f$ on $\Omega$ such that $\left(X_{n}\right)$ and $\left(f \circ \tau^{n}\right)$ have the same joint distributions if and only if the distribution of $\left(X_{n}\right)$ is stationary. We propose to study the corresponding problem for gmp-transformations.

As is shown in [K] and [LW], the map $f \rightarrow f \circ \tau$ can be extended to gmptransformations by putting, for real-valued measurable $f$,

$$
T_{\varphi} f(\omega)=\sup \{t \in \mathbb{R}: \omega \in \phi(\{f>t\})\} .
$$

* Research partially supported by NSF Grant DMS-89-01267 and a Fulbright Research Grant.

** This research was done during a visit of the second author at the Georgia Institute of Technology, Atlanta. This visit was supported by the Deutsche Forschungsgemeinschaft. 
$T_{\varphi}$ is nonlinear, in general, and satisfies $\left\{T_{\varphi} f \geq t\right\}=\phi(\{f \geq t\})$. If $\phi=\phi_{\tau}$, then $T_{\varphi} f=f \circ \tau$.

We say that a process $\left(X_{n}\right)_{n \geq 0}$ can be represented by a gmp-transformation if there exists a gmp-transformation $\phi$ on a suitable probability space $(\Omega, \mathcal{A}, \mu)$, and a measurable $f$ such that the sequence $\left(T_{\varphi}^{n} f\right)$ has the same joint distribution as $\left(X_{n}\right)$. Our question now is: Which processes can be represented by a gmp-transformation?

We shall consider the following conditions:

$\left(\mathrm{M}_{n}\right)$ (Monotonicity condition for intersections): For all $n \in \mathbb{N}$ and all $t_{0}, \ldots t_{n-1} \in \mathbb{R}$

$$
P\left(\bigcap_{i=0}^{n-1}\left\{X_{i} \geq t_{i}\right\}\right) \leq P\left(\bigcap_{i=0}^{n-1}\left\{X_{i+1} \geq t_{i}\right\}\right) .
$$

$\left(\mathrm{M}_{U}\right)$ (Monotonicity condition for unions): For all $n \in \mathbb{N}$ and all $t_{0}, \ldots t_{n-1} \in \mathbb{R}$

$$
P\left(\bigcup_{i=0}^{n-1}\left\{X_{i} \geq t_{i}\right\}\right) \geq P\left(\bigcup_{i=0}^{n-1}\left\{X_{i+1} \geq t_{i}\right\}\right) .
$$

We shall see below that these monotonicity conditions are necessary for a process to permit the representation by a gmp-transformation. This is a fairly simple consequence of (1.1) and (1.2). We do not know if all processes satisfying these two conditions can be represented by a gmp-transformation. In this direction we obtain only a very special result: If $\left(X_{0}, X_{1}, X_{2}, X_{3}\right)$ assumes only two values and the monotonicity conditions above hold, a representation for these 4-dimensional marginals is obtained. Even this special case requires a considerable argument. Our proof relies on a general method for constructing gmp-transformations which satisfy certain priority rules. This is presented in Section 2, and it seems of independent interest. Progress on the main problem seems to require new methods for the construction of gmp-transformations. It is clear that the class of gmp-transformations is very rich, but at present only few methods of construction are available.

Any gmp-transformation $\phi$ induces a transformation, also denoted by $\phi$, in the measure algebra $\overline{\mathcal{A}}$ obtained from $\mathcal{A}$ by identifying sets that differ only by null sets. It will be convenient to look mainly at the measure algebra, and we shall be satisfied with constructing $\phi$ on it.

\section{§2. Construction of gmp-Transformations by Priority Rules}

In $[\mathrm{K}]$, examples of gmp-transformations on a finite set $\Omega$ with counting measure were obtained by prescribing certain priorities for the points of the space. We now introduce an extension of this idea to general measure spaces.

Theorem 2.1. Let $(\Omega, \mathcal{A}, \mu)$ be a probability space supporting an ergodic invertible measure preserving transformation $\tau$. Let $\left\{\Omega_{1}, \Omega_{2}, \ldots\right\}$ be a partition of $\Omega$ into finitely many or countably many (disjoint) measurable sets. For each $i=1,2, \ldots$, let $\left\{\Omega_{i 1}, \Omega_{i 2}, \ldots\right\}$ be a measurable partition of $\Omega$. Put $\Omega^{0}=\emptyset, \Omega^{i}:=\bigcup_{k=1}^{i} \Omega_{k}$, 
$\Omega_{i}^{j}:=\bigcup_{k=1}^{j} \Omega_{i k}$, and for any $A \in \mathcal{A}, A^{i}=A \cap \Omega^{i}$. There exists a gmp-transformation $\phi$ with the following property: For any $i \geq 1$ and $j \geq 2$ and any $A \in \mathcal{A}$ if

$$
\mu\left(\left(\Omega_{i j} \cap \phi\left(A^{i}\right)\right) \backslash \phi\left(A^{i-1}\right)\right)>0
$$

then

$$
\mu\left(\Omega_{i}^{j-1} \backslash \phi\left(A^{i}\right)\right)=0
$$

Remark. The heuristic meaning of the property above is the following: We imagine that the image $\phi(A)$ is constructed by first mapping $A \cap \Omega_{1}$, then mapping $A \cap \Omega_{2}$, then $A \cap \Omega_{3}$, etc. So, after $i$ steps one has constructed $\phi\left(A^{i}\right)$. If the part of the image constructed in the $i^{\text {th }}$ step contains a non-null subset of $\Omega_{i j}$, then $\Omega_{i}^{j-1}$ must already be filled up, i.e., $\Omega_{i}^{j-1}$ must be ( $\bmod$ null sets) a subset of $\phi\left(A^{i}\right)$. In other words, when a subset of $\Omega_{i}$ is mapped, one maps as much as possible into $\Omega_{i 1}$, then as much as possible into $\Omega_{i 2}$ etc. The sets $\Omega_{i j}$ are called priority sets for $\Omega_{i}$.

Proof: Step 1. (Construction of $\phi\left(A \cap \Omega_{1}\right)$ ). This step consists of a countable sequence of substeps. In the first substep, one tries to map as much as possible from $A \cap \Omega_{1}$ into $\Omega_{11}$, in the second substep one maps as much as possible from the remaining part of $A \cap \Omega_{1}$ into $\Omega_{12}$, etc. Formally, the first substep of Step 1 is defined by an inductive procedure. Set

$$
B_{0}^{1}=A^{1} \cap \Omega_{11}=R_{0}^{1} .
$$

We shall have $\phi\left(R_{0}^{1}\right)=B_{0}^{1}$. (On $\Omega_{1} \cap \Omega_{11}, \phi$ is the identity map.) We imagine that we have two copies of $\Omega$, the original space and a second copy for the images. We paint that part of $A$ which has already been mapped red and the image blue in the second copy. So at this point, $R_{0}^{1}$ is painted red and $B_{0}^{1}$ blue. Now, we take the part of $A^{1}$ which is not yet painted and try to map it into the unpainted part of $\Omega_{11}$ by $\tau$. Formally

$$
\begin{aligned}
& B_{1}^{1}=\tau\left(A^{1} \backslash R_{0}^{1}\right) \cap\left(\Omega_{11} \backslash B_{0}^{1}\right) \\
& R_{1}^{1}=\tau^{-1} B_{1}^{1} .
\end{aligned}
$$

Then we continue with $\tau^{2}$ in the same way:

$$
\begin{aligned}
& B_{2}^{1}=\tau^{2}\left(A^{1} \backslash\left(R_{0}^{1} \cup R_{1}^{1}\right)\right) \cap\left(\Omega_{11} \backslash\left(B_{0}^{1} \cup B_{1}^{1}\right)\right) \\
& R_{2}^{1}=\tau^{-2} B_{2}^{1} .
\end{aligned}
$$

It is clear how to continue.

Suppose that a subset $F$ of positive measure of $\Omega_{11}$ is not painted blue in this procedure. As $\tau$ is ergodic,

$$
F^{*}=\bigcup_{k=0}^{\infty} \tau^{-k} F
$$

is almost all of $\Omega$. Any point in $F^{*} \cap A^{1}$ must be painted red. In this case almost all of $A^{1}$ has already been mapped into $\Omega_{11}$ and therefore will not be mapped into $\Omega_{12} \cup \Omega_{13} \cup \ldots$ in the future steps.

If no such $F$ exists, $\Omega_{11}$ is painted blue and we perform the second substep of Step 1 , replacing $\Omega_{11}$ by $\Omega_{12}$ and $A^{1}$ by $A^{1} \backslash \bigcup_{m=0}^{\infty} R_{m}^{1}$, the unpainted part of $A^{1}$. If we write 
$B_{m 1}^{1}$ and $R_{m 1}^{1}$ for the sets $B_{m}^{1}$ and $R_{m}^{1}$ constructed in the first substep above, the next sequence of steps could start with

$$
A_{2}^{1}=A^{1} \backslash \bigcup_{m=0}^{\infty} R_{m 1}^{1}
$$

and

Next put

$$
B_{02}^{1}=A_{2}^{1} \cap \Omega_{12}=R_{02}^{1}
$$

$$
\begin{aligned}
& B_{12}^{1}=\tau\left(A_{2}^{1} \backslash R_{02}^{1}\right) \cap\left(\Omega_{12} \backslash B_{02}^{1}\right) \\
& R_{12}^{1}=\tau^{-1} B_{12}^{1},
\end{aligned}
$$

etc. One continues as above. In this substep either almost all of $\Omega_{12}$ is painted blue or almost all of $A_{2}^{1}$ is painted red. In the latter case, $\phi\left(A^{1}\right)$ shall be the union of all blue sets constructed so far; in the former case one must now repeat the construction with $\Omega_{13}$ and the unpainted part of $A^{1}$.

As $\left(\Omega_{11}, \Omega_{12}, \ldots\right)$ is a partition of $\Omega$, almost all of $A^{1}$ will be painted red after finitely many or denumerably many substeps. $\phi\left(A^{1}\right)$ shall be the union of all sets painted blue in this procedure.

Step 2. Step 2 is just the same as Step 1 except that $A \cap \Omega_{1}$ is now replaced by $A \cap \Omega_{2}$ and the sets $\Omega_{11}, \Omega_{12}, \ldots$ are replaced by

$$
\Omega_{21} \backslash \phi\left(A^{1}\right), \Omega_{22} \backslash \phi\left(A^{1}\right), \ldots
$$

(The set $\phi\left(A^{1}\right)$ has already been painted in Step 1 and is no longer available as an image.) If $B_{01}^{2}, B_{11}^{2}, B_{21}^{2}, \ldots$ is the family of all blue sets constructed in the first substep of Step $2, B_{02}^{2}, B_{12}^{2}, \ldots$ the family of all blue sets constructed in the second substep of Step 2, etc. let $\phi\left(A \cap \Omega^{2}\right)$ be the union of $\phi\left(A \cap \Omega_{1}\right)$ and all these blue sets found in Step 2.

In Step 3 repeat the construction with $A \cap \Omega_{3}$ and with the sets $\Omega_{31} \backslash \phi\left(A \cap \Omega^{2}\right), \Omega_{32} \backslash \phi\left(A \cap \Omega^{2}\right)$, etc. Finally $\phi(A)$ is the union of all blue sets constructed in Steps $1,2,3, \ldots$ It is clear that $\mu(\phi(A))=\mu(A)$ since $A$ is $\bmod \mu$ the disjoint union of red sets $R_{m i}^{j}$ and $\phi(A)$ the corresponding disjoint union of blue sets $B_{m i}^{j}=\tau^{m} R_{m i}^{j}$. If $\widetilde{A} \supset A$, then, at each stage of the construction, the total blue set for $\widetilde{A}$ is at least as large as the corresponding blue set for $A$. The unpainted part of $\widetilde{A}$ is, at each stage, at least as large as the unpainted part of $A$ in the corresponding step of the construction of $\phi(A)$. Hence $\phi(\widetilde{A}) \supset \phi(A)$. By the construction, if $\mu\left(\left(\Omega_{i j} \cap \phi\left(A^{i}\right)\right) \backslash \phi\left(A^{i-1}\right)>0\right.$, then this means that a subset of $\Omega_{i j}$ of positive measure was painted blue in Step i. This happens only if almost all of $\Omega_{i}^{j-1}$ was painted blue before. Hence the gmp-transformation $\phi$ has the desired property.

Remark. If $\mu\left(A \cap \Omega_{i}\right) \leq \mu\left(\Omega_{i}^{j} \backslash \phi\left(A^{i-1}\right)\right)$, then $\phi\left(A^{i}\right) \backslash \phi\left(A^{i-1}\right)$ is, $\bmod \mu$, a subset of $\Omega_{i}^{j}$. (If $\mu\left(\Omega_{i k} \cap\left(\phi\left(A^{i}\right) \backslash \phi\left(A^{i-1}\right)\right)\right)$ is positive for some $k>j$, then $\Omega_{i}^{j} \backslash \phi\left(A^{i-1}\right)$ must have been painted blue.) 


\section{§3. Representation of Processes by gmp-Transformations}

Theorem 3.1. The conditions $\left(M_{\cap}\right)$ and $\left(M_{U}\right)$ are necessary for a process $\left(X_{m}\right)$ to admit a representation by a gmp-transformation.

Proof. Assume $\left(X_{m}\right)$ admits a representation on a probability space $(\Omega, \mathcal{A}, \mu)$. Then there exists a measurable $f$ and a gmp-transformation $\phi$ such that $\left(T_{\varphi}^{m} f\right)$ has the same joint distribution as $\left(X_{m}\right)$. Using $\left\{T_{\varphi}^{k} f \geq t\right\}=\phi^{k}(\{f \geq t\})(k \geq 0)$ and (1.1) we obtain

$$
\begin{aligned}
P\left(X_{0} \geq t_{0}, \ldots, X_{n-1} \geq t_{n-1}\right) & =\mu\left(\bigcap_{i=0}^{n-1} \phi^{i}\left(\left\{f \geq t_{i}\right\}\right)\right) \\
& =\mu\left(\phi \bigcap_{i=0}^{n-1} \phi^{i}\left(\left\{f \geq t_{i}\right\}\right)\right) \\
& \leq \mu\left(\bigcap_{i=0}^{n-1} \phi^{i+1}\left(\left\{f \geq t_{i}\right\}\right)\right) \\
& =P\left(X_{1} \geq t_{0}, \ldots, X_{n} \geq t_{n-1}\right) .
\end{aligned}
$$

The symmetric argument with (1.2) shows that also $\left(M_{\cup}\right)$ is necessary.

We do not know if the combined conditions $\left(M_{\cap}\right)$ and $\left(M_{\cup}\right)$ are sufficient. It even seems hard to answer this problem when the process takes only two values, say 0 and 1 , and we ask only that for any fixed $n$ there exists a $\phi$ and $f$ such that $\left(X_{i}\right)_{i=0}^{n}$ and $\left(T_{\varphi}^{i} f\right)_{i=0}^{n}$ have the same joint distribution. In this case, $f$ is an indicator function $f=1_{A_{0}}$, and we have $T_{\varphi}^{k} f=1_{\phi^{k}\left(A_{0}\right)}$.

Most of the remainder of this paper will be devoted to showing that the combination $(M)$ of $\left(M_{\cup}\right)$ and $\left(M_{\cap}\right)$ is sufficient for this subproblem when $n \leq 3$.

Let $(\Omega, \mathcal{A}, \mu)$ be a nonatomic probability space supporting an ergodic invertible measure preserving transformation $\tau$ in $\Omega$. E.g., $(\Omega, \mathcal{A}, \mu)$ is the unit interval with Lebesgue measure. Clearly, we can assume $\left(\Omega^{\prime}, \mathcal{A}^{\prime}, P\right)=(\Omega, \mathcal{A}, \mu)$ replacing the original process $\left(X_{i}\right)_{i=0}^{n}$ by a process with the same distribution defined on $(\Omega, \mathcal{A}, \mu)$.

Let us reformulate the conditions $\left(M_{\cap}\right)$ and $\left(M_{\cup}\right)$ for 0 -1-valued processes $X_{i}=$ $1_{A_{i}}(i=0, \ldots, n)$ on $(\Omega, \mathcal{A}, \mu)$. For any nonempty subset $I=\left\{i_{1}, i_{2}, \ldots, i_{k}\right\}$ of $\{0,1, \ldots, n-1\}$ put

$$
A_{\cap I}:=\bigcap_{\nu=1}^{k} A_{i_{\nu}}, \quad A_{\cup I}:=\bigcup_{\nu=1}^{k} A_{i_{\nu}}
$$

and

$$
A_{\cap I+1}:=\bigcap_{\nu=1}^{k} A_{i_{\nu}+1}, \quad A_{\cup I+1}:=\bigcup_{\nu=1}^{k} A_{i_{\nu}+1} .
$$


It is an exercise to show that $(M)$ is equivalent to the condition $\left(M^{\prime}\right)$ that for all $n \in \mathbb{N}$, both

$$
\mu\left(A_{\cap I}\right) \leq \mu\left(A_{\cap I+1}\right) \text { for all } I \subset\{0, \ldots, n-1\}
$$

and

$$
\mu\left(A_{\cup I}\right) \geq \mu\left(A_{\cup I+1}\right) \text { for all } I \subset\{0, \ldots, n-1\} .
$$

Note that $\left(M^{\prime}\right)$ implies $\mu\left(A_{i}\right)=\mu\left(A_{i+1}\right)(0 \leq i \leq n-1)$ by taking $I=\{i\}$.

By Theorem 3.1, $\left(M^{\prime}\right)$ is necessary for the existence of a gmp-transformation $\phi$ with $\phi^{i}\left(A_{0}\right)=A_{i}(1 \leq i \leq n)$. It seems natural to conjecture that $\left(M^{\prime}\right)$ is also sufficient, but we can prove this only for $n \leq 3$. For larger $n$, the present approach gets extremely involved, and a new, possibly more canonical construction seems desirable. For $n \leq 3$, we actually prove a stronger result.

Let $B_{0}, B_{1}, \ldots$ be measurable sets. Define $B_{\cap I}$ and $B_{\cup I}$ just like $A_{\cap I}$ and $A_{\cup I}$.

Theorem 3.2. Let $(\Omega, \mathcal{A}, \mu)$ be a nonatomic probability space supporting an invertible ergodic measure preserving transformation $\tau$ in $\Omega$. Let $A_{0}, \ldots, A_{n-1}$ and $B_{0}, \ldots, B_{n-1}$ be measurable sets, $n \leq 3$. The condition $\left(M^{\prime \prime}\right)$ that

$$
\mu\left(A_{\cap I}\right) \leq \mu\left(B_{\cap I}\right)
$$

and

$$
\mu\left(A_{\cup I}\right) \geq \mu\left(B_{\cup I}\right)
$$

hold for all $I \subset\{0, \ldots, n-1\}$ is necessary and sufficient for the existence of a gmptransformation $\phi$ with $B_{i}=\phi\left(A_{i}\right)(i=0, \ldots, n-1)$.

Proof. The necessity holds for all $n$ and is easily established using (1.1) and (1.2).

With the help of Theorem 2.1, the proof of the sufficiency is easy for $n=2$ : Map $\Omega_{1}:=A_{0} \cap A_{1}$ into $\Omega_{11}:=B_{0} \cap B_{1}$. Then map $\Omega_{2}:=A_{0} \backslash A_{1}$ into $\Omega_{21}:=B_{0}$, then $\Omega_{3}=A_{1} \backslash A_{0}$ into $\Omega_{31}:=B_{1}$, and finally $\Omega_{4}:=\left(A_{0} \cup A_{1}\right)^{c}$ into $\Omega_{41}=\Omega$. We leave the details as an exercise. Note that $\left(M^{\prime \prime}\right)$ implies $\mu\left(A_{0}\right)=\mu\left(B_{0}\right), \mu\left(A_{1}\right)=\mu\left(B_{1}\right)$, and $\mu\left(A_{0} \cap A_{1}\right) \leq \mu\left(B_{0} \cap B_{1}\right)$.

$n=3$ : We consider the partition of $\Omega$ induced by the sets $A_{0}, A_{1}, A_{2}$. It consists of the sets

$$
\begin{array}{ll}
\Sigma_{1}:=A_{0} \cap A_{1} \cap A_{2} & \Sigma_{2}:=A_{0} \cap A_{1} \cap A_{2}^{c} \\
\Sigma_{3}:=A_{0} \cap A_{1}^{c} \cap A_{2} & \Sigma_{4}:=A_{0} \cap A_{1}^{c} \cap A_{2}^{c} \\
\Sigma_{5}:=A_{0}^{c} \cap A_{1} \cap A_{2} & \Sigma_{6}:=A_{0}^{c} \cap A_{1} \cap A_{2}^{c} \\
\Sigma_{7}:=A_{0}^{c} \cap A_{1}^{c} \cap A_{2} & \Sigma_{8}:=A_{0}^{c} \cap A_{1}^{c} \cap A_{2}^{c} .
\end{array}
$$

The partition $\left\{\Sigma_{1}^{*}, \Sigma_{2}^{*}, \ldots, \Sigma_{8}^{*}\right\}$ induced by the sets $B_{0}, B_{1}, B_{2}$ is defined in exactly the same way: $\Sigma_{1}^{*}:=B_{0} \cap B_{1} \cap B_{2}$, etc. Set

$$
\delta_{i}=\mu\left(\Sigma_{i}^{*}\right)-\mu\left(\Sigma_{i}\right) \quad(i=1, \ldots, 8) .
$$


(The actual sizes of the sets are less important than the differences $\delta_{i}$.) Assume $\left(M^{\prime \prime}\right)$ holds. In terms of the $\delta_{i}$ 's this means that the following identities and inequalities are satisfied:

$$
\begin{aligned}
\delta_{1}+\delta_{2}+\delta_{3}+\delta_{4} & =0 \\
\delta_{1}+\delta_{2}+\delta_{5}+\delta_{6} & =0 \\
\delta_{1}+\delta_{3}+\delta_{5}+\delta_{7} & =0 \\
\delta_{1} & \geq 0 \\
\delta_{1}+\delta_{2} & \geq 0 \\
\delta_{1}+\delta_{3} & \geq 0 \\
\delta_{1}+\delta_{5} & \geq 0 \\
\delta_{2}+\delta_{3}+\delta_{5}+2 \delta_{1} & \geq 0
\end{aligned}
$$

(The last inequality follows from $\sum_{i=1}^{7} \delta_{i} \leq 0$ by subtracting the three identities in (3.1).)

The construction of $\phi$ shall be based on Theorem 2.1. The priorities will depend on the $\delta$ 's. In all cases, we shall start by mapping $\Sigma_{1}$ into $\Sigma_{1}^{*}$ because $\Sigma_{1}$ is a subset of all $A_{i}$, so that $\phi\left(\Sigma_{1}\right)$ must be a subset of all $B_{i}$.

If we specify priority sets $\Omega_{k 1}, \Omega_{k 2}, \ldots, \Omega_{k m}$ which do not cover all of $\Omega$, this shall mean that there is one additional set $\Omega_{k, m+1}$ which is the complement of the union of $\Omega_{k 1}, \ldots, \Omega_{k m}$.

Case 1. $\delta_{3} \geq 0$. In this case, we start by mapping those $\Sigma_{i}$ which are contained in $A_{1}$. $\Sigma_{3}^{*}$ will be big enough to recieve all of $\Sigma_{3}$, and no part of $\Sigma_{1}^{*}$ will be needed as image of $\Sigma_{3}$. Therefore we can map $\Sigma_{2}$ into $\Sigma_{1}^{*} \cup \Sigma_{2}^{*}$. Possibly, $\Sigma_{5}$ is too big to be mapped into $\Sigma_{5}^{*}$, but we shall see that no harm is done mapping the surplus into $\Sigma_{6}^{*}$.

Formally, set

$$
\begin{gathered}
\Omega_{1}=\Sigma_{1}, \quad \Omega_{11}=\Sigma_{1}^{*} \\
\Omega_{2}=\Sigma_{2}, \quad \Omega_{21}=\Sigma_{2}^{*}, \quad \Omega_{22}=\Sigma_{1}^{*}
\end{gathered}
$$

(so, tacitly, $\Omega_{23}=\Omega \backslash\left(\Omega_{21} \cup \Omega_{22}\right)$.

$$
\begin{aligned}
& \Omega_{3}=\Sigma_{5}, \quad \Omega_{31}=\Sigma_{5}^{*}, \quad \Omega_{32}=\Sigma_{1}^{*}, \quad \Omega_{33}=\Sigma_{6}^{*} \\
& \Omega_{4}=\Sigma_{6}, \quad \Omega_{41}=B_{1}, \quad \Omega_{42}=B_{1}^{c}
\end{aligned}
$$

(By now we know how to map subsets of $A_{1}$.) Next, set

$$
\begin{array}{ll}
\Omega_{5}=\Sigma_{3}, & \Omega_{51}=\Sigma_{3}^{*} \\
\Omega_{6}=\Sigma_{4}, & \Omega_{61}=B_{0} \\
\Omega_{7}=\Sigma_{7}, & \Omega_{71}=B_{2} \\
\Omega_{8}=\Sigma_{8}, & \Omega_{81}=\Omega .
\end{array}
$$

This determines $\phi$. We have to check $\phi\left(A_{i}\right)=B_{i}$ for $i=0,1,2$ :

$(i=0): A_{0}=\Sigma_{1} \cup \Sigma_{2} \cup \Sigma_{3} \cup \Sigma_{4} . \Sigma_{1} \cup \Sigma_{2}$ is mapped into $\Sigma_{1}^{*} \cup \Sigma_{2}^{*}$ since $\delta_{1}+\delta_{2} \geq 0$.

$\Sigma_{3}$ goes into $\Sigma_{3}^{*}$ since $\delta_{3} \geq 0 . \Sigma_{4}$ goes into $B_{0}$ since $\mu\left(A_{0}\right)=\mu\left(B_{0}\right)$. Hence $\phi\left(A_{0}\right)=B_{0}$. $(i=1): \phi\left(A_{1}\right)=B_{1}$ is clear. 
( $i=2$ ): Constructing $\phi\left(A_{2}\right)$, first $\Sigma_{1}$ is mapped into $\Sigma_{1}^{*}$. $A_{2} \cap \Sigma_{2}$ is empty. Therefore, when $A_{2}$ is mapped, the priorities for $\Sigma_{2}$ do not matter. $\Sigma_{5}$ is mapped into $\Sigma_{1}^{*} \cup \Sigma_{5}^{*}$ since no part of this set was consumed by $\phi\left(A_{2} \cap \Sigma_{2}\right)$. Thus, $\Sigma_{5}$ goes into $B_{2}$. $\Sigma_{3}$ goes into $\Sigma_{3}^{*} \subset B_{2}$; finally $\Sigma_{7}$ goes into $B_{2}$ since $\mu\left(B_{2}\right)=\mu\left(A_{2}\right)$. Thus, $\phi\left(A_{2}\right) \subset B_{2}$. As $\mu\left(\phi\left(A_{2}\right)\right)=\mu\left(A_{2}\right)=\mu\left(B_{2}\right)$, we have $\phi\left(A_{2}\right)=\phi\left(B_{2}\right)$.

The cases $\delta_{2} \geq 0$ and $\delta_{5} \geq 0$ are symmetric. Thus, we can assume $\delta_{3}<0$, $\delta_{2}<0, \delta_{5}<0$ in the sequel.

Case 2. $\delta_{1}+\delta_{2}+\delta_{3} \geq 0$. This time, let the sets $\Omega_{i}$ and $\Omega_{i j}$ be defined by

$$
\begin{aligned}
& \Omega_{1}=\Sigma_{1}, \quad \Omega_{11}=\Sigma_{1}^{*} \\
& \Omega_{2}=\Sigma_{2}, \quad \Omega_{21}=\Sigma_{2}^{*}, \quad \Omega_{22}=\Sigma_{1}^{*} \\
& \Omega_{3}=\Sigma_{5}, \quad \Omega_{31}=\Sigma_{5}^{*}, \quad \Omega_{32}=\Sigma_{1}^{*}, \quad \Omega_{33}=\Sigma_{6}^{*} \cup \Sigma_{2}^{*} \\
& \Omega_{4}=\Sigma_{3}, \quad \Omega_{41}=\Sigma_{3}^{*}, \quad \Omega_{42}=\Sigma_{1}^{*}, \quad \Omega_{43}=\Sigma_{5}^{*} \cup \Sigma_{7}^{*} \\
& \Omega_{5}=\Sigma_{4}, \quad \Omega_{51}=B_{0} \\
& \Omega_{6}=\Sigma_{6}, \quad \Omega_{61}=B_{1} \\
& \Omega_{7}=\Sigma_{7}, \quad \Omega_{71}=B_{2} \\
& \Omega_{8}=\Sigma_{8}, \quad \Omega_{81}=\Omega \text {. }
\end{aligned}
$$

We must, again, check $\phi\left(A_{i}\right)=B_{i}(i=0,1,2)$ :

$(i=0): \Sigma_{1} \cup \Sigma_{2}$ is mapped into $\Sigma_{1}^{*} \cup \Sigma_{2}^{*}$ again. Recall that we can assume $\delta_{2}<0$. Thus $\Sigma_{2}^{*}$ is covered by $\phi\left(\Sigma_{2}\right)$. Next, $\Sigma_{3}$ is mapped into $\Sigma_{1}^{*} \cup \Sigma_{3}^{*}$ since $\delta_{1}+\delta_{2}+\delta_{3} \geq 0$ means that there is enough space left over in $\Sigma_{1}^{*} \cup \Sigma_{1}^{*} \cup \Sigma_{3}^{*}$; all this space must actually be in $\Sigma_{1}^{*} \cup \Sigma_{3}^{*}$ because $\Sigma_{2}^{*}$ is already covered. $B_{0}$ is large enough to receive the remaining part $\Sigma_{4}$ of $A_{0}$. Hence $\phi\left(A_{0}\right) \subset B_{0}$; and then $\mu\left(A_{0}\right)=\mu\left(\phi\left(A_{0}\right)\right)=\mu\left(B_{0}\right)$ yields $\phi\left(A_{0}\right)=B_{0}$.

$(i=1)$ : This is even simpler and therefore deleted.

$(i=2): \phi\left(\Sigma_{1} \cup \Sigma_{5}\right)$ fits into $\Sigma_{1}^{*} \cup \Sigma_{5}^{*}$. Then any part of $\Sigma_{3}$ which is not mapped into $\Sigma_{1}^{*} \cup \Sigma_{3}^{*}$ is mapped into $\Sigma_{5}^{*} \cup \Sigma_{7}^{*} \subset B_{0}$. Finally, $\Sigma_{7}$ is mapped into $B_{2}$, too.

The cases $\delta_{1}+\delta_{2}+\delta_{5} \geq 0$ and $\delta_{1}+\delta_{3}+\delta_{5} \geq 0$ are symmetric. Thus it remains to study

Case 3: $\delta_{1}+\delta_{2}+\delta_{3}<0, \delta_{1}+\delta_{2}+\delta_{5}<0, \delta_{1}+\delta_{3}+\delta_{5}<0$. Recall that we can also assume $\delta_{2}<0, \delta_{3}<0, \delta_{5}<0$, and have $\delta_{1}+\delta_{2} \geq 0, \delta_{1}+\delta_{3} \geq 0, \delta_{1}+\delta_{5} \geq 0, \delta_{1}>0$ from (3.2). Let $E$ be a subset of $\Sigma_{1}^{*}$ having measure $\mu(E)=\delta_{1}$, and let $E_{2}, E_{3}, E_{5}$ be three subsets of $E$ with

$$
\mu\left(E_{i}\right)=\left|\delta_{i}\right| \quad(i=2,3,5)
$$

such that no point of $E$ belongs to all three sets $E_{2}, E_{3}, E_{5}$. It is possible to find such subsets since (3.2) holds and

$$
2 \delta_{1}+\delta_{2}+\delta_{3}+\delta_{5} \geq 0
$$

Using $\delta_{1}+\delta_{2}+\delta_{3}<0$, we can assume that $E=E_{2} \cup E_{3}$. (The sets $E_{2}, E_{3}$ need not be disjoint.) 
As $\mu\left(\Sigma_{5}\right)-\mu\left(\Sigma_{5}^{*}\right)=-\delta_{5}=\mu\left(E_{5}\right)$, and as $E_{2} \cap E_{5}$ and $E_{3} \cap E_{5}$ are disjoint, we can find three disjoint subsets $\Sigma_{51}, \Sigma_{52}, \Sigma_{53}$ of $\Sigma_{5}$ such that

$$
\begin{aligned}
& \mu\left(\Sigma_{51}\right)=\mu\left(\Sigma_{5}^{*}\right) \\
& \mu\left(\Sigma_{52}\right)=\mu\left(E_{2} \cap E_{5}\right) \\
& \mu\left(\Sigma_{53}\right)=\mu\left(E_{3} \cap E_{5}\right) .
\end{aligned}
$$

Let $\phi$ be the gmp-transformation obtained by applying Theorem 2.1 with

$$
\begin{aligned}
\Omega_{1}=\Sigma_{1}, & \Omega_{11}=\Sigma_{1}^{*} \backslash E \\
\Omega_{2}=\Sigma_{2}, & \Omega_{21}=\Sigma_{2}^{*}, \quad \Omega_{22}=E_{2} \\
\Omega_{3}=\Sigma_{3}, & \Omega_{31}=\Sigma_{3}^{*}, \quad \Omega_{32}=E_{3}, \quad \Omega_{33}=\Sigma_{4}^{*} \\
\Omega_{4}=\Sigma_{51}, & \Omega_{41}=\Sigma_{5}^{*}, \\
\Omega_{5}=\Sigma_{52}, & \Omega_{51}=E_{2} \cap E_{5}, \quad \Omega_{52}=\Sigma_{6}^{*}, \quad \Omega_{53}=B_{1} \backslash\left(E_{3} \cap E_{5}\right) \\
\Omega_{6}=\Sigma_{53}, & \Omega_{61}=E_{3} \cap E_{5}, \quad \Omega_{62}=\Sigma_{7}^{*}, \quad \Omega_{63}=B_{2} \backslash \Sigma_{7}^{*} \\
\Omega_{7}=\Sigma_{4}, & \Omega_{71}=B_{0} \\
\Omega_{8}=\Sigma_{6}, & \Omega_{81}=B_{1} \\
\Omega_{9}=\Sigma_{7}, & \Omega_{91}=B_{2} \\
\Omega_{10}=\Sigma_{8}, & \Omega_{10,1}=\Omega
\end{aligned}
$$

Again, we check that $\phi\left(A_{i}\right)=B_{i}$ for $i=0,1,2$.

$(i=0)$ : When $\phi\left(A_{0}\right)$ is constructed, first $\Omega_{1} \cap A_{0}=\Sigma_{1}$ goes into $\Sigma_{1}^{*} \backslash E$ and fills it up. Then $\Omega_{2} \cap A_{0}$ is mapped. Part of it goes into $\Sigma_{2}^{*}$ and fills it up, and the surplus goes into $E_{2} \subset \Sigma_{1}^{*}$. Now, $\Sigma_{2}^{*} \cup E_{2}$ is filled up.

Next, $\Omega_{3} \cap A_{0}=\Sigma_{3}$ is mapped. First $\Sigma_{3}^{*}$ is filled, then $E_{3} \backslash E_{2}$ is filled (since $E_{2}$ was full already). The surplus goes into $\Sigma_{4}^{*}$. It fits into this set because $B_{0} \backslash \Sigma_{4}^{*}$ is already filled up at this time.

$\Omega_{4} \cap A_{0}, \Omega_{5} \cap A_{0}$ and $\Omega_{6} \cap A_{0}$ are empty. $\Omega_{7} \cap A_{0}=\Sigma_{4}$. None of this set is mapped to $B_{0} \backslash \Sigma_{4}^{*}$ since that set is full by now. In view of $\mu\left(A_{0}\right)=\mu\left(B_{0}\right)$ there must be just enough space in $\Sigma_{4}^{*}$ left over to receive the image of $\Omega_{7} \cap A_{0}$. Hence $\phi\left(A_{0}\right)=B_{0}$.

$(i=1): \Omega_{1} \cap A_{1}=\Sigma_{1}$ goes into $\Sigma_{1}^{*} \backslash E$. Next, $\Omega_{2} \cap A_{1}=\Sigma_{2}$ goes into $\Sigma_{2}^{*} \cup E_{2}$ and fills it up. $\Omega_{3} \cap A_{1}$ is empty. $\Omega_{4} \cap A_{1}=\Sigma_{51}$ goes into $\Sigma_{5}^{*}$ and fills it. $\Omega_{5} \cap A_{1}=\Sigma_{52}$. The first priority for this set would be $E_{2} \cap E_{5}$, but all of $E_{2}$ is already occupied. So, this set is mapped into $\Sigma_{6}^{*}$ and into $B_{1} \backslash\left(E_{3} \cap E_{5}\right)$. There is enough space in these sets since they form the remainder of $B_{1}$ except for $E_{3} \cap E_{5}$ and the subset $\Sigma_{53}$ of $A_{1}$ remains to be mapped. (Recall that $\mu\left(\Sigma_{53}\right)=\mu\left(E_{3} \cap E_{5}\right)$.) Thus, $\Sigma_{52}$ is mapped into $B_{1} \backslash\left(E_{3} \cap E_{5}\right)$. Next, $\Omega_{6} \cap A_{1}=\Sigma_{53}$ is mapped, and it gets its first priority since $E_{3} \cap E_{5}$ was kept in reserve. The remaining part of $A_{1}$ is $\Sigma_{6}$. It fits into $B_{1}$. Hence, $\phi\left(A_{1}\right)=B_{1}$.

$(i=2): \Omega_{1} \cap A_{2}=\Sigma_{1}$ is mapped onto $\Sigma_{1}^{*} \backslash E . \Omega_{2} \cap A_{2}$ is empty. $\Omega_{3} \cap A_{2}=\Sigma_{3}$ is mapped to $\Sigma_{3}^{*} \cup E_{3}$ and fills this set. $\Omega_{4} \cap A_{2}=\Sigma_{51}$ fits into $\Sigma_{5}^{*} \subset B_{2} . \Omega_{5} \cap A_{2}=\Sigma_{52}$ fits into $E_{2} \cap E_{5}$ which is still unoccupied since $\Sigma_{3}$ was mapped to $\Sigma_{3}^{*} \cup E_{3}$ and this set is disjoint from $E_{2} \cap E_{5} . \Omega_{6} \cap A_{2}=\Sigma_{53}$ has $E_{3} \cap E_{5}$ as first priority. This set is occuppied, but the next priorities are in $B_{2} . \Omega_{7} \cap A_{2}$ and $\Omega_{8} \cap A_{2}$ are empty. $\Omega_{9} \cap A_{2}=\Sigma_{7}$ goes into $B_{2}$. Thus $\phi\left(A_{2}\right)=B_{2}$. 
Remarks: (1) Clearly, only the measure algebra corresponding to $(\Omega, \mathcal{A}, \mu)$ matters. Thus, we can delete the assumption of existence of $\tau$ if $(\Omega, \mathcal{A}, \mu)$ is nonatomic and $\mathcal{A}$ countably generated.

(2) For $n=2$, the present conditions are equivalent to the requirement that $\mu\left(B_{i}\right)=$ $\mu\left(A_{i}\right)(i=0, \ldots, n-1)$, and

$$
\mu\left(A_{\cap I}\right) \leq \mu\left(B_{\cap I}\right)
$$

for all $I \subset\{0, \ldots, n-1\}$. For $n=3$ however, the condition that $\mu\left(A_{\cup I}\right) \geq \mu\left(B_{\cup I}\right)$ for all $I$ cannot be replaced by the condition that $\mu\left(B_{i}\right)=\mu\left(A_{i}\right)$ for all $i$, even when $B_{i}=A_{i+1}$. The following sets $A_{i}(i=0, \ldots, 3)$ in $\Omega=[0,1]$ with Lebesgue measure $\mu$ can serve as an example: $A_{0}:=[0, .4], A_{1}:=[.2, .6], A_{2}:=[0, .2] \cup[.4, .6], A_{3}:=[.1, .3] \cup[.4, .5] \cup[.6, .7]$. We have $\mu\left(A_{i}\right)=.4$ for all $i, \mu\left(A_{i} \cap A_{j}\right)=.2$ for all $i \neq j$ and $\mu\left(A_{0} \cap A_{1} \cap A_{2}\right)=0<$ $\mu\left(A_{1} \cap A_{2} \cap A_{3}\right)=.1$. However, $\mu\left(A_{0} \cup A_{1} \cup A_{2}\right)=.6<\mu\left(A_{1} \cup A_{2} \cup A_{3}\right)=.7$.

\section{References}

[K] Krengel, U. Generalized measure preserving transformations, Proceed. Conf. on Almost Everywhere Convergence, Ohio State Univ. 1988, Academic Press (1989), 215-235.

[LW] Lin, M. and R. Wittmann. Pointwise ergodic theorems for certain order preserving mappings in $L^{1}$, to appear in Proceed. Conf. on Almost Everywhere Convergence II, Evanston, 1989. 